\title{
DESENVOLVIMENTO PONDERAL, BIOMETRIA TESTICULAR E QUALIDADE SEMINAL DE TOUROS NELORE (Bos taurus indicus) CRIADOS EXTENSIVAMENTE NA REGIÃO CENTRO-OESTE DO BRASIL
}

\author{
(Body growth, testicular biometry and seminal quality of Nelore \\ (Bos Taurus indicus) bulls extensively raised in Middle West of Brazil) \\ VIU, M.A.O.'; MAGNABOSCO, C.U. ${ }^{2}$ FERRAZ, H.T. ${ }^{3}$; GAMBARINI, M.L.4; \\ OLIVEIRA FILHO, B.D. ${ }^{4}$; LOPES, D.T. ${ }^{3}$; VIU, A.M.F. ${ }^{5}$
}

\begin{abstract}
1Professor da Escola de Veterinária - UFG/Jataí, Laboratório de Reprodução Animal - (64) 3632-1510, marcoviu@yahoo.com.br;

${ }^{2}$ Pesquisador EMBRAPA Cerrados - Bolsista CNPq; ${ }^{3}$ Alunos de Pós-Graduação em Ciência Animal - EV/UFG. Bolsista CAPES/CNPq; ${ }^{4}$ Professores da Escola de Veterinária - UFG/Goiânia; ${ }^{5}$ Professora da Escola de Ciências Biológicas - UFG/Jataí.
\end{abstract}

\begin{abstract}
RESUMO - Com o objetivo de avaliar características testiculares e seminais de touros Nelore criados extensivamente em uma região de clima tropical de savana, 271 animais foram divididos em quatro classes de idade (C1, 17 a 20 meses; C2, 21 a 24 meses; C3, 25 a 30 meses e C4, 31 a 36 meses) e submetidos à avaliação andrológica. O peso vivo (PV) foi obtido imediatamente antes da colheita do sêmen, realizandose a aferição do comprimento (COMP) e largura (LARG) dos testículos, assim como da circunferência escrotal (CE). O volume (VT) e peso testicular (PT) foram calculados, e a qualidade seminal avaliada por meio das características físicas: motilidade progressiva (MOT); vigor (VIG) e turbilhonamento (TUR); e morfológicas: defeitos maiores (DMA); menores (DME) e totais (DT) do ejaculado. Para determinação da forma testicular (FT) utilizou-se a razão LARG/COMP, classificando-se em: longa; longa-moderada; longa-oval; oval-esférica e esférica. Houve correlação positiva de moderada intensidade $(P<0,01)$ entre $P V$ e CE, $P T$ e VT. As características PV, CE, PT e VT mostraram correlação negativa de moderada intensidade $(P<0,01)$ com DMA, DME e DT. Predominaram as formas alongadas dos testículos, com correlação positiva $(\mathrm{P}<0,01)$ entre $\mathrm{PV}, \mathrm{CE}$ e $\mathrm{FT}$, além de correlação negativa $(P<0,05)$ entre $P V, C E, F T$ e patologias espermáticas. O formato esférico não esteve presente em nenhuma das classes de idade. Conclui-se que os fatores relacionados à biometria testicular (BT) aumentam com o ganho de peso em touros Nelore de 17 a 36 meses de idade e que, além da CE, o VT, o PT e a FT podem ser parâmetros úteis para a seleção de reprodutores Nelore.
\end{abstract}

Palavras-chave: andrologia; morfologia espermática; termorregulação.

ABSTRACT - With the objective to evaluate the morphological testicular characteristics an seminal quality of Nelore bulls raised extensively in a region where predominates the tropical of savannah climate, 271 animals were divided in four age classes: (C 1) of 17 to 20 months; (C2) of 21 to 24 months; (C3) of 25 to 30 months; (C4) of 31 to 36 months and submitted to breeding soundness evaluation (BSE). The animals were weighted immediately before BSE and testicular length (COMP) and width (LARG), as well as the scrotal circumference (CE) were taken. The testicular volume (VT) and testicular weight (PT) were calculated, and seminal quality evaluated through physical characteristics as progressive motility (MOT), vigor (VIG) and gross activity (TUR); morphological characteristics were reported as major defects (DMA), minor (DME) and total (DT). Testicular form (FT) was determined according the reason $L A R G / C O M P$, and classified in: long; longmoderate; long-oval; oval-spherical and spherical. There was a moderate and positive correlation $(P<0,01)$ between PV and CE, PT and VT. The traits PV, CE, PT and VT showed a moderate and negative $(P<0,01)$ with DMA, DME and DT. The alongated forms of the testicles had predominated, with positive correlation $(P<0.01)$ with $\mathrm{PV}, \mathrm{CE}$ and FT. However, the correlation $(\mathrm{P}<0,05)$ of $\mathrm{PV}, \mathrm{CE}, \mathrm{FT}$ with spermatic morphology was negative. The spherical format was not present in none of the age class. It was possible to conclude that factors related to testicular biometrics (BT) increase with weight gain in Nelore bulls from 17 to 36 months of age and not only CE, but VT, PT and FT could also be useful parameters to selection of Nelore bulls.

Key-words: andrology; sperm morphology; thermoregulation.

\section{Introdução}

A circunferência escrotal (CE) é uma mensuração facilmente obtida, com alta repetibilidade entre 
avaliadores, além de ser uma característica que possui herdabilidade moderada a alta (DIAS et al., 2003), correlacionada com o ganho de peso (VALENTIM et al., 2002), produção espermática (PALASZ et al., 1994; SANTOS et al., 1998; KASTELIC et al., 2001) e qualidade seminal dos touros (SILVA et al., 2002; VALENTIM et al., 2002). No entanto, segundo BAILEY et al. (1998), a CE é uma medida indireta da massa testicular, que não considera a variação individual na forma dos testículos e na qual a espessura da parede da bolsa escrotal é erroneamente somada. Sendo assim, de acordo com estes mesmos autores, comprimento e largura testicular podem ser boas ferramentas para auxiliar na estimativa da fertilidade do reprodutor, por meio do uso destas medidas para o cálculo do volume (VT) e peso testiculares (PT). Touros com menor CE e testículos mais alongados podem ter maior VT e/ou PT do que animais que apresentam o formato ovóide a esférico, com produção espermática comparável àqueles de maior diâmetro (BAILEY et al., 1996).

Segundo BAILEY et al. (1996), freqüentemente os reprodutores bovinos apresentam a forma ovóide dos testículos, porém a variação para formas alongadas é comum, principalmente em zebuínos (UNANIAN et al., 2000), podendo causar a ocorrência de CE's menos expressivas do que em taurinos. Essa característica pode ser extremamente vantajosa, ainda mais considerando que testículos menos esféricos apresentam maior superfície de contato com o meio ambiente, facilitando a termorregulação, além de possibilitar a distribuição mais uniforme dos vasos sangüíneos e tecido espermático, favorecendo os padrões seminais (BAILEY et al., 1996).

Poucos estudos correlacionaram as variações normais das formas testiculares (FT) à produção e qualidade espermática, sendo que, na literatura disponível, existem poucos relatos do estudo da influência das FT sobre as características do sêmen de Bos taurus indicus. O objetivo deste estudo foi avaliar as correlações entre o peso vivo (PV), as medidas de biometria testicular (CE, VT e PT) e as diferentes FT com a qualidade seminal, determinadas por meio da análise dos aspectos físicos e morfológicos do ejaculado, em touros Nelore de 17 a 36 meses de idade criados extensivamente na região Centro-Oeste do Brasil.

\section{Material e Métodos}

O conjunto de dados utilizado no presente estudo foi obtido de 271 avaliações andrológicas realizadas em touros Nelore PO criados extensivamente na região sudoeste do Estado de Goiás. Os animais estudados pertenciam ao mesmo rebanho, mantidos em sistema extensivo de criação, em pastagem de capim-marandú (Brachiaria brizantha Hochst ex. A. Rich Stapf), recebendo suplementação mineral no período chuvoso e complementação com mistura protéico-energética (20\% PB e $70 \%$ NDT) durante o período seco.

Os animais foram distribuídos em quatro classes de acordo com a idade: Classe 1 ( $C 1, n=109), 17$ a 20 meses; Classe 2 (C2, n=43), 21 a 24 meses; Classe 3 (C3, $n=96), 25$ a 30 meses; e Classe 4 (C4, n=23), 31 a 36 meses. Após a identificação, os touros foram pesados e submetidos ao exame clínico geral e do sistema genital, avaliando-se a CE com o auxílio de fita métrica no maior diâmetro da bolsa escrotal. As medidas de COMP e LARG dos testículos foram obtidas com o uso de paquímetro. O VT e o PT foram calculados segundo BAILEY et al. (1998). Para determinação da forma dos testículos, utilizou-se a razão LARG/COMP, segundo metodologia descrita por UNANIAN et al. (2000), classificando-se em: longa (razão 0,5); longamoderada (razão de 0,51 a 0,625); longa-oval (razão de 0,626 a 0,750); oval-esférica (razão de 0,751 a 0,875 ); e esférica (razão $\geq 0,875$ ).

As amostras de sêmen foram obtidas por eletroejaculação, sendo uma gota do ejaculado imediatamente analisada em microscópio óptico para avaliação do TUR, MOT e VIG. Uma alíquota foi depositada em tubos plásticos contendo solução formol salina tamponada, para a avaliação da morfologia espermática em câmara úmida, com microscópio de contraste de fase em aumento de 1000 vezes. As patologias foram classificadas em defeitos maiores (DMA), menores (DME) e totais (DT), segundo o preconizado pelo Colégio Brasileiro de Reprodução Animal (CBRA, 1998).

As análises de crítica e consistência dos dados foram realizadas por meio do procedimento UNIVARIATE (SAS, 2000), constatando-se distribuição normal de probabilidade e homogeneidade de variância dos erros experimentais para as características morfológicas do ejaculado, porém as características físicas (MOT, VIG e TUR) passaram por transformações radiciais (raiz quadrada de $x+1$ ), onde $x$ é a característica estudada, para que os pressupostos de normalidade e homogeneidade fossem obedecidos. Procedeu-se então a análise de variância utilizando-se o procedimento General Linear Model (SAS, 2000). O coeficiente de correlação de Pearson foi calculado utilizando-se o procedimento CORR (SAS, 2000), determinando a intensidade da associação entre as características estudadas.

\section{Resultados e Discussão}

Os dados expostos na TABELA 1 mostram que, entre os animais da $\mathrm{C} 1$ a média para $\mathrm{CE}$ foi superior àquelas relatadas por SILVA et al. (2002) em touros Nelore de 18 meses de idade $(27,22 \mathrm{~cm})$. VALENTIM et al. (2002), trabalhando com touros Nelore de 20 meses, também verificaram média inferior $(27,40 \mathrm{~cm})$ à observada neste estudo. 
Desenvolvimento ponderal, biometria testicular e qualidade seminal de touros nelore...

TABELA 1 - MÉDIA ( $\mu$ ) E DESVIO-PADRÃO (DP), NAS DIVERSAS CLASSES DE IDADE, DAS VARIÁVEIS CIRCUNFERÊNCIA ESCROTAL (CE), PESO MÉDIO DOS TESTÍCULOS (PT), VOLUME MÉDIO DOS TESTÍCULOS (VT), MOTILIDADE PROGRESSIVA (MOT), VIGOR (VIG), TURBILHONAMENTO (TUR), DEFEITOS MAIORES (DMA), DEFEITOS MENORES (DME), DEFEITOS TOTAIS (DT) E PESO DO TOURO (PV), JATAÍ-GO, 2006.

\begin{tabular}{lcccc}
\hline \multicolumn{1}{c}{ Variáveis } & $\begin{array}{c}\text { Classe } 1 \\
\mu \pm D P\end{array}$ & $\begin{array}{c}\text { Classe } 2 \\
\mu \pm D P\end{array}$ & $\begin{array}{c}\text { Classe } 3 \\
\mu \pm D P\end{array}$ & $\begin{array}{c}\text { Classe } 4 \\
\mu \pm D P\end{array}$ \\
\hline CE $(\mathrm{cm})$ & $30,02 \pm 3,03^{\mathrm{a}}$ & $33,19 \pm 2,84^{\mathrm{b}}$ & $33,97 \pm 2,40^{\mathrm{b}}$ & $33,87 \pm 2,72^{\mathrm{b}}$ \\
PT (g) & $202,07 \pm 43,44^{\mathrm{a}}$ & $317,47 \pm 109,34^{\mathrm{b}}$ & $429,42 \pm 198,68^{\mathrm{c}}$ & $336,87 \pm 76,46^{\mathrm{d}}$ \\
VT (cm $\left.{ }^{3}\right)$ & $191,22 \pm 41,11^{\mathrm{a}}$ & $300,43 \pm 103,47^{\mathrm{b}}$ & $406,37 \pm 188,01^{\mathrm{c}}$ & $318,79 \pm 72,35^{\mathrm{d}}$ \\
MOT (\%) & $75,32 \pm 5,58$ & $76,74 \pm 5,44$ & $74,74 \pm 5,91$ & $75,00 \pm 9,65$ \\
VIG (1-5) & $3,60 \pm 0,62^{\mathrm{a}}$ & $3,90 \pm 0,65^{\mathrm{b}}$ & $3,80 \pm 0,72^{\mathrm{b}}$ & $4,00 \pm 0,74^{\mathrm{b}}$ \\
TUR (1-5) & $3,06 \pm 0,95^{\mathrm{a}}$ & $3,74 \pm 0,73^{\mathrm{b}}$ & $3,46 \pm 0,75^{\mathrm{c}}$ & $3,57 \pm 0,79^{\mathrm{cd}}$ \\
DMA (\%) & $20,66 \pm 10,89^{\mathrm{a}}$ & $5,93 \pm 2,76^{\mathrm{b}}$ & $6,73 \pm 4,30^{\mathrm{bc}}$ & $4,57 \pm 3,03^{\mathrm{bd}}$ \\
DME (\%) & $7,56 \pm 4,16^{\mathrm{a}}$ & $4,26 \pm 4,02^{\mathrm{b}}$ & $4,29 \pm 2,79^{\mathrm{b}}$ & $3,91 \pm 2,90^{\mathrm{b}}$ \\
DT (\%) & $28,22 \pm 12,40^{\mathrm{a}}$ & $10,19 \pm 5,96^{\mathrm{b}}$ & $11,02 \pm 5,89^{\mathrm{b}}$ & $8,48 \pm 4,12^{\mathrm{b}}$ \\
PV (kg) & $416,79 \pm 37,48^{\mathrm{a}}$ & $428,53 \pm 46,42^{\mathrm{ab}}$ & $519,45 \pm 68,46^{\mathrm{c}}$ & $565,17 \pm 46,29^{\mathrm{d}}$ \\
\hline
\end{tabular}

Letras diferentes na mesma linha diferiram estatisticamente ao nível de $5 \%$ de probabilidade, pelo teste t.

O mesmo ocorreu para a C2, cuja CE média foi superior àquelas observadas por estes autores em touros Nelore de 24 meses $(29,20 \mathrm{~cm})$. Para SILVA et al. (2002), a seleção para CE em touros de até 18 meses de idade é um critério importante na escolha dos futuros reprodutores. Este é um dos principais critérios de seleção dos animais destinados à reprodução no rebanho estudado, e isto pode explicar a superioridade dos dados aqui relatados.

Houve correlação positiva de moderada intensidade $(P<0,01)$ entre PV e CE (TABELA 2). VALENTIM et al. (2002) encontraram correlações semelhantes entre PV e CE. Esses resultados indicam que parte dos genes envolvidos na expressão dessas características é comum. Por sua vez, PV, CE, PT e VT mostraram correlação negativa de moderada intensidade $(P<0,01)$ com as características morfológicas do sêmen (DMA, DME e DT), indicando que, com o avançar da idade, os animais ganham peso, aumentam as medidas testiculares e melhoram os aspectos morfológicos do ejaculado. TROCÓNIZ et al. (1991) verificaram maior número de patologias espermáticas em animais de 13 a 15 meses de idade do que naqueles entre 22 e 24 meses, corroborando com os dados aqui apresentados. VALENTIM et al. (2002) relataram correlações negativas entre CE e DT, assim como PALASZ et al. (1994), que obtiveram correlação positiva entre CE e percentagem de espermatozóides normais. Estes mesmos autores relataram correlações positivas entre CE e PT, CE e produção diária de espermatozóides e entre CE e as reservas epididimárias de espermatozóides.

TABELA 2 - COEFICIENTES DE CORRELAÇÃO DE PEARSON (r) ENTRE O PESO DO ANIMAL (PV), AS MEDIDAS DE BIOMETRIA TESTICULAR: CIRCUNFERÊNCIA ESCROTAL (CE); PESO MÉDIO DOS TESTÍCULOS (PT); VOLUME MÉDIO DOS TESTÍCULOS (VT); FORMAS TESTICULARES (FT), AS CARACTERÍSTICAS FÍSICAS DO SÊMEN: MOTILIDADE PROGRESSIVA (MOT); VIGOR (VIG) E TURBILHONAMENTO (TUR), E AS MORFOLOGICAS: DEFEITOS MAIORES (DMA); MENORES (DME) E TOTAIS (DT), JATAÍ-GO, 2006.

\begin{tabular}{|c|c|c|c|c|c|c|c|}
\hline \multicolumn{8}{|c|}{ Características do ejaculado x Características Biométricas e Peso } \\
\hline & PV & MOT & VIG & TUR & DMA & DME & DT \\
\hline PV & 1,00 & $-0,03$ & $0,16^{*}$ & $0,14^{* *}$ & $-0,37^{*}$ & $-0,31^{*}$ & $-0,40^{*}$ \\
\hline CE & $0,41^{*}$ & 0,05 & $0,15^{*}$ & $0,25^{*}$ & $-0,45^{*}$ & $-0,30^{*}$ & $-0,46^{*}$ \\
\hline PT & $0,51^{*}$ & $-0,04$ & 0,11 & 0,09 & $-0,27^{*}$ & $-0,20^{*}$ & $-0,28^{*}$ \\
\hline VT & $0,51^{*}$ & $-0,04$ & 0,11 & 0,09 & $-0,27^{*}$ & $-0,20^{*}$ & $-0,28^{*}$ \\
\hline FT & $0,44^{*}$ & $-0,06$ & 0,08 & 0,06 & $-0,27^{*}$ & $-0,13^{*}$ & $-0,26^{*}$ \\
\hline \multicolumn{8}{|c|}{ Características biométricas dos testículos e Peso } \\
\hline & PV & & & PT & \multirow{2}{*}{\multicolumn{2}{|c|}{$\begin{array}{c}\mathrm{VT} \\
0,30^{* *}\end{array}$}} & FT \\
\hline PV & 1,00 & \multicolumn{2}{|c|}{0,02} & $0,30 *$ & & & 0,36 ** \\
\hline CE & 0,02 & \multirow{2}{*}{\multicolumn{2}{|c|}{1,00}} & $0,40^{* *}$ & \multicolumn{2}{|c|}{0,40} & $0,32^{* *}$ \\
\hline PT & $0,30 *$ & & & 1,00 & \multicolumn{2}{|c|}{$1,00^{* *}$} & $0,43^{* *}$ \\
\hline VT & $0,30 *$ & \multicolumn{2}{|c|}{$0,40^{* * *}$} & $1,00^{* *}$ & \multicolumn{2}{|c|}{1,00} & $0,43^{* *}$ \\
\hline FT & $0,36 *$ & \multicolumn{2}{|c|}{$0.32^{* *}$} & $0,43^{* *}$ & \multicolumn{2}{|c|}{$0,43^{* *}$} & 1,00 \\
\hline
\end{tabular}

*Nível de significância de $1 \%(P<0,01)$; ${ }^{* \star}$ Nível de significância de $5 \%(P<0,05)$. 
A influência do PV, CE, PT, VT e FT sobre as características físicas do ejaculado (MOT, VIG e TUR) foi muito variável, com correlações positivas e negativas de baixa intensidade e a maioria delas sem significância (TABELA2), o que pode estar relacionado ao fato destas variáveis serem de avaliação subjetiva. VALENTIM et al. (2002) encontraram correlações positivas de baixa intensidade entre CE e MOT e entre CE e VIG, quando da avaliação de touros Nelore com idade entre 20 e 24 meses.

Ainda na TABELA 2, são mostradas as correlações entre as $\mathrm{FT}$ e os defeitos espermáticos. A correlação negativa $(\mathrm{P}<0,05)$ entre $\mathrm{PV}, \mathrm{CE}, \mathrm{FT}$ e as patologias espermáticas sinaliza que, conforme os animais ganham peso, a CE aumenta e a FT tende a passar do formato longo para ovalado, resultando em melhoria da qualidade espermática, representada pela diminuição dos DMA, DME e DT.

De acordo com os dados expostos na TABELA 3, entre os animais da $\mathrm{C} 1$ predominou a FT classificada como longa-moderada, com nenhum dos animais apresentando as formas oval-esférica e esférica. UNANIAN et al. (2000) verificaram freqüência semelhante em touros Nelore de 18 meses. Dentre os animais da $\mathrm{C} 2$, também houve predominância da forma longa-moderada, e novamente, a forma esférica de testículos não esteve presente em nenhum animal. Nos animais da C3 e C4 a FT mais freqüentemente observada foi a longa-oval, e nenhum animal mostrou testículos de formato esférico. O teste Qui-quadrado mostrou diferença $(P<0,01)$ entre as formas testiculares nas diferentes classes de idade.

Analisando-se a distribuição das FT nas classes de idade, observa-se que com o aumento da idade o formato dos testículos vai se modificando, tornando-se gradativamente mais ovalado. Fato semelhante foi descrito por UNANIAN et al. (2000), que observaram a mudança de forma dos testículos em $73 \%$ dos tourinhos Nelore dos 12 aos 18 meses de idade. Estes mesmos autores relataram a predominância das formas alongadas e a ausência da forma esférica, assim como verificado neste estudo. BAILEY et al. (1996) observaram que animais com testículos mais alongados apresentavam maior concentração de espermatozóides no ejaculado que aqueles de formato testicular mais esférico. Devido ao exposto anteriormente e analisandose a TABELA de freqüência das FT (TABELA 3), podese considerar os animais da $\mathrm{C} 1$ classificados como portadores de testículos longo-ovóides, como sendo de alto valor fenotípico quando se considera a precocidade sexual, uma vez que estes indivíduos possuem boa CE para a idade (TABELA 1) e forma testicular mais adequada à produção espermática (BAILEY et al., 1996).

TABELA 3 - FREQÜÊNCIAS (\%) DAS FORMAS DOS TESTÍCULOS (RAZÃO LARG/COMP) NAS DIFERENTES CLASSES DE IDADE, EM TOUROS NELORE, JATAÍ-GO, 2006.

\begin{tabular}{lcccc}
\hline & \multicolumn{4}{c}{ Freqüências (\%) } \\
Forma do Testículo & Classe 1 & Classe 2 & Classe 3 & Classe 4 \\
\hline Longa & 15,60 & 23,26 & 1,04 & 0 \\
Longa-moderada & 69,72 & 65,12 & 23,96 & 34,78 \\
Longa-oval & 14,68 & 9,30 & 53,13 & 60,87 \\
Oval-esférica & 0 & 2,33 & 21,88 & 4,35 \\
Esférica & 0 & 0 & 0 & 0 \\
\hline
\end{tabular}

\section{Conclusões}

Com base nos dados avaliados, conclui-se que, nas condições ambientais e de criação em que este estudo foi conduzido, as características relacionadas à biometria testicular aumentam com o ganho de peso em touros Nelore de 17 a 36 meses de idade e que, além da CE, o VT, o PT e a FT podem ser parâmetros úteis na seleção de reprodutores Nelore.

\section{Referências}

BAILEY, T.L.; MONKE, D.; HUDSON, R.S.; WOLFE, D.F.; CARSON, R.L.; RIDDELL, M.G. Testicular shape and its relationship to sperm production in mature Holstein bulls. Theriogenology, New York, v.46, p.881-887, 1996.

BAILEY, T.L.; HUDSON, R.S.; POWE, T.A.; RIDDELL, M.G.; WOLFE, D.F.; CARSON, R.L. Caliper and ultrasonographic measurements of bovine testicles and a mathematical formula for determining testicular volume and weight in vivo. Theriogenology, New York, v.49, p.581-594, 1998.

CBRA. Manual para exame andrológico e avaliação de sêmen animal. Colégio Brasileiro de Reprodução Animal. 2. Ed. - Belo Horizonte: CBRA, 1998. 49p.

DIAS, L.T.; EL FARRO, L.; ALBUQUERQUE, L.C. Estimativas de herdabilidade para perímetro escrotal de animais da raça Nelore. Revista Brasileira de Zootecnia, Viçosa, v.32, n.6, p.1878-1882, 2003.

KASTELIC, J.P.; COOK, R.B.; PIERSON, R.A.; COULTER, G.H. Relationships among scrotal and testicular characteristics, sperm production, and seminal quality in 129 beef bulls. The Canadian Journal of Veterinary Research, Montreal, v.65, p.111-115, 2001.

PALASZ, A.T.; CATES, W.F.; BARTH, A.D.; MAPLETOFT, R.J. The relationship between scrotal circumference and quantitative testicular traits in yearling beef bulls. Theriogenology, New York, v.42, n.4, p.715-726, 1994. 
Desenvolvimento ponderal, biometria testicular e qualidade seminal de touros nelore...

SANTOS, M.D.; TORRES, C.A.A.; GUIMARÃES, J.D.; PEREIRA, J.C.; MACHADO, G.V. Sêmen e circunferência escrotal de touros zebu alimentados com dois níveis de concentrado e lipídeos. Revista Brasileira de Zootecnia, Viçosa, v.27, n.3, p.627-632, 1998.

SAS - User's Guide: Statistics. 5.ed. Cary: SAS Institute, 2000. 1028p.

SILVA, A.E.D.F.; UNANIAN, M.M.; CORDEIRO, C.M.T.; FREITAS, A.R. Relação da circunferência escrotal e parâmetros de qualidade do sêmen em touros da raça Nelore, PO. Revista Brasileira de Zootecnia, Viçosa, v.31, n.3, p.1157-1165, 2002.

TROCÓNIZ, J.F.; BELTRÁN, J.; BASTIDAS, H.; LARREAL, H.; BASTIDAS, P. Testicular development, body weight changes, puberty and semen traits of growing Guzerat and Nellore bulls. Theriogenology, New York, v.35, n.4, p.815-826, 1991.

UNANIAN, M.M.; SILVA, A.E.D.F.; McMANUS, C.; CARDOSO, E.P. Características biométricas testiculares para avaliação de touros zebuínos da raça Nelore. Revista Brasileira de Zootecnia, Viçosa, v.29, n.1, p.136-144, 2000.

VALENTIM, R.; ARRUDA, R.P.; BARNABÉ, R.C.; ALENCAR, M.M. Biometria testicular de touros Nelore e touros cruzados europeu-Nelore aos 20 e 24 meses de idade. Brazilian Journal of Veterinary Research and Animal Science, São Paulo, v.39, n.3, p.113-120, 2002.

Recebido para publicação: $\quad$ 23/08/2006 Aprovado: 